\title{
Comunicación
}

\section{EIMERIOSIS EN CRÍAS DE ALPACAS: ESTUDIO ANÁTOMO HISTOPATOLÓGICO}

\author{
César Palacios E. ${ }^{1}$, Luis Tabacchi N. ${ }^{2}$, Alfonso Chavera C. ${ }^{2}$, Teresa López U. ${ }^{3}$, \\ Gilberto Santillán A. ${ }^{2}$, Nieves Sandoval Ch. ${ }^{2}$, Danilo Pezo C. ${ }^{4}$ y Rosa Perales C. ${ }^{2}$
}

\section{Abstract}

\begin{abstract}
The death associated with diarrhea of 7 newborn alpacas from the Maranganí-Cuzco community was studied. The animals showed signs of reddish watery diarrhea, dehydration, anorexia, and weakness. The gross lesions in the small intestine mucosa were reddish, augmented and necrotic, with severe hiperemia and gas. They showed small white foci of $0.2 \mathrm{~cm}$ diameter located at the final part of yeyuno and ileon. Two types of oocysts were detected. The first was piriform-shape of 90 microns (Eimeria macusaniensis) and the second, elipsoidal-shape of 30 microns (Emeria lamae). Microscopic lesions presented an acute, diffuse, and severe catarrhal necrotic enteritis with hiperplasia of Lieberkun cripts, and thickness or shortness of the villi, with moderate to severe corionic infiltration of eosinophils, linfocites and plasmacites, along with numerous endogenous forms: schizonts, microgamonts, macrogamonts, and nonsporulated oocysts at level of the crypts and villi.
\end{abstract}

Key words: new born alpaca, diarrhea, Eimeria, coccidia

Las enfermedades parasitarias constituyen el principal problema sanitario en las explotaciones de camélidos sudamericanos. La principal enfermedad parasitaria que cursa con mortalidad de crías es la eimeriosis (Ameghino y De Martin, 1991). Hasta el momento se han descrito 5 tipos de eimeria que afectan a las alpacas: E. alpacae, E. lamae, E. punoensis (Guerrero, 1967) que afectan el epitelio de las vellosidades (Guerrero et al., 1967), la E. macusaniensis (Guerrero et al., 1971) que afecta las glándulas crípticas (Gue- rrero et al., 1967), y la E. ivitaensis (Leguía y Casas, 1998) cuya patogenia es desconocida. La coccidiosis se presenta, generalmente, de forma subclínica, con o sin diarrea leve. En casos clínicos el signo más característico es una leve diarrea sanguinolenta y fétida (Ameghino y De Martin, 1971; Rojas, 1990). Se ha observado que bajo condiciones de campo y en forma experimental, la E. macusaniensis y la E. lamae, constituyen la asociación más patógena (Guerrero et al., 1970).

\footnotetext{
I Práctica privada

2 Laboratorio de Histo-Patología Animal, FMV-UNMSM

3 Laboratorio de Microbiología y Parasitología Veterinaria, FMV-UNMSM

4 Estación Experimental del Centro de Investigaciones IVITA-Maranganí, FMV-UNMSM
} 
Aún cuando se reconoce que la prevalencia de eimeriosis en alpacas es elevada, se desconoce su importancia en el complejo diarreico y en la mortalidad de crías. Aunque hay trabajos que describen las lesiones causadas por E. macusaniensis (Guerrero et al., 1967; Hodgin et al., 1984; Rosadio y Ameghino, 1989) y por E. lamae (Guerrero et al., 1967, 1970), éstas no describen las respuestas celulares ni diferencian las porciones intestinales afectadas, y sólo describen someramente las medidas de algunas formas endógenas.

En una comunidad alpaquera de Maranganí, Cusco, murieron 38 crías de alpacas de 4 a 5 meses de edad entre julio y agosto del 2002. De éstas, siete presentaron signos clínicos de diarrea acuosa a sanguinolenta, deshidratación, anorexia, y debilidad compatibles con una severa eimeriosis.

Se hicieron exámenes microscópicos del intestino delgado e intestino grueso. Se recolectaron segmentos de intestino delgado (duodeno, yeyuno e ileon) e intestino grueso (ciego, colon y recto) y se realizaron raspados de la mucosa del yeyuno e ileon para su observación al microscopio. Se tomaron muestras de cada segmento intestinal en formalina al $15 \%$ para su evaluación histopatológica. El yeyuno se dividió en tres subsecciones: inicial, medio y final. Los cortes fueron de 5 a 6 $\mu$ de grosor, coloreándose con HematoxilinaEosina, Azul de Toluidina, Giemsa y Tricrómico de Shorr.

Al examen microscópico se halló una duodenitis necrótica. El yeyuno tenía la mucosa engrosada y exfoliada con leve hiperemia y puntos blanquecinos en el tramo final. La mucosa del ileon se observó necrótica y severamente hiperémica, con puntuaciones blanquecinas. El ciego severamente dilatado y la mucosa exfoliada con enrojecimiento difuso. El colon estaba severamente dilatado y la mucosa muy hiperémica; en algunos casos, acompañado de hiperplasia de las placas de Séller (hasta $4 \mathrm{~cm}$ de diámetro).
En el examen microscópico de los raspados pertenecientes a las puntuaciones blanquecinas de la mucosa se apreciaron ooquistes sin esporular de Eimeria macusaniensis y de E. lamae. En el análisis histopatológico se identificaron las siguientes formas endógenas (las medidas se tomaron en una muestra de 10 especímenes):

- $\quad$ El esquizonte de 161.4 x $145 \mu$.

- La macrogametogonia con tres estadios de desarrollo. El macrogamonte joven con 25.8 x $25.8 \mu$, el macrogamonte casi maduro con 72.5 x $64.4 \mu$, y el macrogamonte maduro con $17.5 \mu$ de diámetro.

- La microgametogonia con dos estadios de desarrollo. El microgamonte inmaduro con $139.8 \times 121.3 \mu$ y el microgamonte maduro con 204.4 x $174.6 \mu$.

Los ooquistes inmaduros eran de forma piriforme. La E. macusaniensis con 73 x 51.7 $\mu$, la E. lamae con $28.8 \times 22.5 \mu$. Por otro lado, la E. ivitaensis tenía forma elipsoidal con medidas de $58.3 \times 45.9 \mu$, rodeadas por una cápsula de 4 a $4.5 \mu$ de grosor.

Los cambios histopatológicos causados por E. macusaniensis en el intestino delgado eran de carácter subagudo a crónico, caracterizado por un acortamiento y fusión de vellosidades, acompañado de una severa denudación epitelial con completa exposición de la lámina basal. El corion se encontraba de moderado a severamente infiltrado por linfocitos, plasmocitos, eosinófilos y neutrófilos; y la lámina basal estaba erosionada en algunas zonas dejando escapar al lumen células inflamatorias y eritrocitos. La hiperemia fue más marcada en la zona media apical de las vellosidades, acompañada de un moderado a severo edema con dilatación de quilíferos. Además, se apreciaba ligera a moderada proliferación de tejido conectivo en el corion de algunas vellosidades, con infiltración discreta de mononucleares. Las criptas mostraban una severa hiperplasia, con células hipertrofiadas, de núcleo voluminoso e hipocromático. 


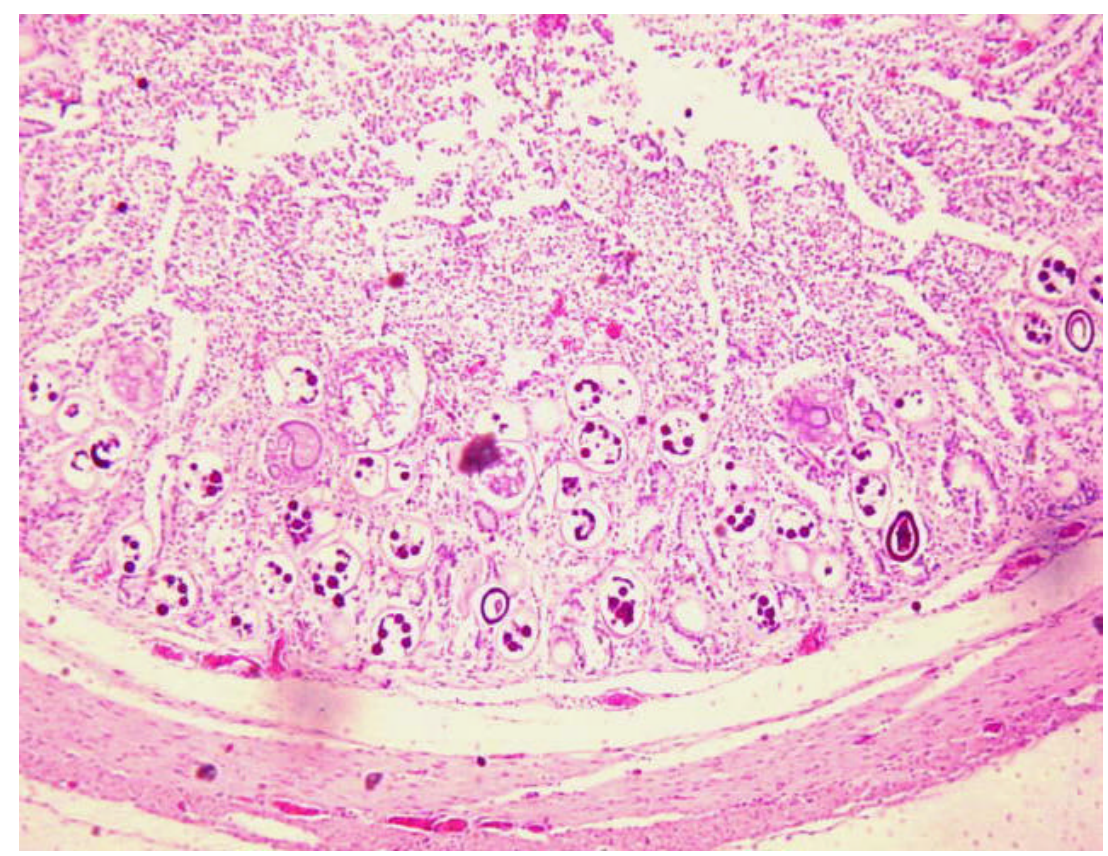

Figura 1. Corte de intestino delgado mostrando la presencia de E. macusanensis y E. ivitaensis a nivel de las glándulas de la cripta (H-E, 25x)

La reacción inflamatoria pericriptal fue predominantemente mononuclear, entre macrófagos linfoplasmocitos con escasos eosinófilos. La muscular de la mucosa se mostraba interrumpida en algunas zonas, acompañada con una leve a moderada vacuolización. En algunas zonas se apreciaban macrogamontes enquistados, algunos con procesos degenerativos. La submucosa presentaba una moderada a severa hiperemia asociada de un moderado a severo edema y moderada infiltración perivascular de mononucleares. La muscular se acompañaba de un moderado a severo edema intra y pericelular, acompañado de moderada hiperemia. La serosa estaba aparentemente normal.

En lesiones asociadas con E. ivitaensis, los cambios a nivel de vellosidad mostraban una severa necrosis (Fig. 1), con el corion completamente invadido por mononucleares. Las figuras de mitosis en las criptas eran severas; llegándose a formar agregaciones de cromatina como prolongaciones en los núcleos. La infiltración de neutrófilos en las crip- tas fue más severa que en los casos donde sólo había E. macusaniensis, y también fue mayor la presencia de sincitios celulares y glándulas de rueda de carreta. En las lesiones asociadas con E. lamae, las vellosidades severamente acortadas eran masivamente infiltradas por eosinófilos acompañadas de una severa hiperemia, y masiva denudación epitelial, llegando algunas veces a erosionar la membrana basal, dejando escapar células inflamatorias y eritrocitos.

El intestino grueso se caracterizaba por lesiones de curso agudo. La mucosa del ciego presentaba moderada hiperemia, acompañada de una severa denudación epitelial con exposición de la membrana basal, acompañado de un moderado a severo edema. Las criptas moderadamente hiperplásicas. La submucosa con leve a moderada hiperemia acompañada de leve a moderado edema; en algunos casos con discreta proliferación de folículos linfoides con leve edema. La muscular con leve a moderado edema peri e intracelular. La serosa estaba aparentemente normal. El colon presentaba cambios semejantes al ciego. 
En el presente estudio se pudo verificar que las formas endógenas de E. macusaniensis no se limitan al yeyuno e ileon como lo señalan algunos autores (Guerrero et al., 1967; Hodgin et al., 1984; Rosadio y Ameghino, 1989), sino que también pueden localizarse en el ciego y colon ascendente, al igual que lo reportado en bovinos (Friend y Stockdale, 1980; Stockdale, 1977), ovinos (Arguello, 1999) y caprinos (Kanyari, 1990; Viera et al., 1997). Las lesiones observadas son de tipo necrotizante exfoliatiava, al igual que la producida por la E. falciformis (Mesfin et al., 1987) a diferencia de otros tipos de eimerias como E. bovis (Friend y Stockdale, 1990) y E. zuernii (Stockdale, 1977), que son diftéricas y en E. ninakolyakimoviae (Viera et al., 1997) que es hemorrágica necrótica.

Las lesiones en las criptas por las formas endógenas de E. macusaniensis y $E$. ivitaensis son similares a las que se aprecian en infecciones naturales y experimentales por E. bovis (Friend y Stockdale, 1990) y E. zuiernii (Stockdale, 1977). En la E. lamae, las células epiteliales se descaman perdiéndose en el lumen intestinal y los capilares dilatados son expuestos junto con la membrana basal erosionada, dejando escapar eritrocitos y células inflamatorias. Otras eimerias hacen el mismo daño, pero generalmente acompañadas de grandes cantidades de fibrina como en la E. zuernii (Stockdale, 1977).

Los gamontes encontrados a nivel de la muscular de la mucosa también se han observado en E. parva (Arguello, 1999) y la presencia de esquizontes en los quilíferos del intestino delgado se ha reportado en varias eimerias; una de ellas es la E. bovis (Arguello, 1999; Friend y Stockdale, 1980). Algo que es importante mencionar, es que a pesar que la E. macusaniensis es uno de las eimerias mas grandes que afectan a los mamíferos, sus formas endógenas no toman dimensiones impresionantes, como sucede en otras, como por ejemplo, la E. candralis cuyos esquizontes llegan a medir hasta 280 micras (Arguello, 1999).

Los cambios histológicos del yeyuno e ileon en el epitelio de la cripta de disociación celular (glándulas de rueda de carreta), sincitios celulares en las glándulas y células gigantes fagocíticas en el corion, se han reportado en E. bovis (Friend y Stockdale, 1980) y las glándulas fenestradas se han descrito en E. zuernii (Stockdale, 1977). En general, se observa una moderada respuesta celular pericriptal en las glándulas que contienen las formas endógenas protozoales. Muchas de las células vistas en el corion no pueden ser reconocidas, pudiendo ser algunas de ellas células de regeneración de las criptas como se ha descrito en otros casos (Stockdale, 1977), y algunas pueden ser fibroblastos pericriptales que comienzan a hacerse prominentes cuando hay colapso de criptas (Mesfin et al., 1987). Sin embargo, en las lesiones por E. macusaniensis y $E$. ivitaensis se aprecian en las criptas hiperplásicas una hipertrofia celular acompañada de figuras de mitosis que llegan a mostrar cromosomas enormemente alargados, similares a lo que se reportan en células parasitadas por E. apsheronica (Kanyari, 1990). El edema visto en la mucosa y la dilatación de quilíferos puede deberse al mecanismo obstructivo de las diferentes formas endógenas protozoales como se observa en la E. bovis (Friend y Stockdale, 1980).

En conclusión, las lesiones patológicas por coccidiosis en alpacas incluyen destrucción epitelial, edema, inflamación y necrosis, acompañada muchas veces de hiperplasia glandular. Las lesiones fueron más severas en intestino delgado a nivel del yeyuno medio y final. Las formas endógenas de $E$. macusaniensis se encontraron tanto en el intestino delgado como en el ciego y rama ascendente del colon, en tanto que las otras eimerias se concentraron en el intestino delgado. La E. ivitanesis parasita las glándulas junto con la E. macusaniensis. 


\section{Agradecimientos}

Los autores expresan su agradecimiento al Ing. Teodoro Huiza y a los Dres. Wilber García, Víctor Leyva, Armando González y Walter Braga por su decidido apoyo y colaboración para el desarrollo del presente estudio.

\section{Literatura Citada}

1. Ameghino, E.; J. De Martín. 1991. Coccidiosis. En: Mortalidad de crías de alpaca. p 71-112. Centro de Investigación IVITA-UNMSM. Lima.

2. Arguello, H. 1999. Coccidiosis. En: Parasitología veterinaria. p 195-211. Ed. Mac Graw Hill-Interamericana. España.

3. Friend, S.; P. Stockdale. 1980. Experimental Eimeria bovis infections in calves: A histopathological study. Can. J. Comp. Med. 44: 129-140.

4. Guerrero, C. 1967. Cocidia (Protozoo: eimerida) of the Alpaca Lama pacos. J. Parasitol. 14: 613-616.

5. Guerrero, C; J. Hernández; J. Alva. 1967. Coccidiosis en alpacas. Rev. Fac. Med. Vet. UNMSM, Perú 21: 59-68.

6. Guerrero, C.; J. Alva; G. Leguía; H. Bazalar. 1970. Prevalencia de coccidia en alpacas, Lama pacos. Bol. Extraord. 4: 84-90.

7. Guerrero, C; J. Alva; H. Bazalar; L. Tabacchi. 1970. Infección experimental de alpacas con Eimeria lamae . Rev. Fac. Med. Vet. UNMSM, Perú 21: 59-68.
8. Guerrero, C; J. Hernández; H. Bazalar; J. Alva J. 1971. Eimeria macusaniensis (Protozoo: Eimeridae) of the alpaca Lama pacos. J. Parasitol. 18: 162-163.

9. Hodgin, C.; V. Schillhon; R. Fayer. 1984. Leptospirosis and coccidial infection in a guanaco. J. Am. Vet. Med. Assoc. 11: 1442-1444.

10. Kanyari, P. 1990. Eimeria apsheronica in the goat: endogenous development and host celular response. Int. J. Parasitol. 20: 625-630.

11. Leguía, G.; E. Casas. 1998. Eimeria ivitaensis (Protozoa: Eimeridae) en alpacas Lama pacos. Rev. Per. Parasitol. 13: 59-61.

12. Mesfin, G.; J. Bellamy; P. Atockdale. 1987. The pathological changes caused by Eimeria falciformis var. pragensis in mice. Can. J. Com. Med. 42: 496-510.

13. Rojas, M. 1990. Eimeriosis. En: Parasitismo de los rumiantes domésticos. $p$ 301-310. Ed. Maijosa. Lima, Perú.

14. Rosadio, R.; E. Ameghino. 1989. Coccidiosis en alpacas neonatas. Resúmenes XII Reunión Científica Anual APPA. Lima, Perú. p 100.

15. Stockdale, P. 1977. The pathogenesis of the lesions produced by Eimeria zuernii in calves. Can. J. Med. Comp. 41: 338-344.

16. Viera, L.; J. Lima; J. Rosa. 1997. Development of Eimeria ninakohlyakimovae Yakimoff y Rastigaieff, 1930 Emend. Levine, 1961 in experimentally infected goats (Capra hercus). J. Parasitol. 83: 1015-1018. 